\title{
Extinction and PAH intensity variations across the HII region IRAS 12063-6259
}

\author{
David Stock $^{\star a}$, Els Peeters ${ }^{a b}$, Xander Tielens $^{c}$, Jackie Otaguro ${ }^{a}$ and Arjan Bik ${ }^{d}$ \\ ${ }^{a}$ Department of Physics and Astronomy, University of Western Ontario, Canada \\ ${ }^{b}$ SETI Institute, Mountain View, CA, USA \\ ${ }^{c}$ Leiden Observatory, Leiden University, Netherlands \\ ${ }^{d}$ MPIA, Heidelberg, Germany \\ E-mail: dstock4@uwo.ca
}

The spatial variations in polycyclic aromatic hydrocarbon (PAH) band intensities are generally attributed to variations of the physical conditions in the environment hosting the emitting PAH molecules. However, in recent years, it has been suggested that such variations are caused mainly by extinction. To resolve this question, we have obtained near-infrared (NIR), mid-infrared (MIR) and radio observations of the compact HII region IRAS 12063-6259. We use these data to construct multiple independent extinction maps and to measure the main PAH feature intensities (6.2, 7.7, 8.6 and $11.2 \mu \mathrm{m}$ ). Three extinction maps are derived: the first using the NIR hydrogen lines and case B recombination theory; the second combining the NIR data, radio data and case B recombination; and the third making use of the Spitzer/IRS MIR observations to measure the 9.8 $\mu \mathrm{m}$ silicate absorption feature intensity using the Spoon method and PAHFIT. We conclude that different areas of IRAS 12063-6259 possess markedly different extinction properties, with some regions displaying both silicate absorption and corresponding NIR extinction, and other regions displaying NIR extinction and no corresponding silicate absorption. While such breakdowns of the relationship between the NIR extinction and the silicate absorption strength have been observed in molecular clouds, they have never been observed for HII regions. We then compare the PAH intensity variations in the Spitzer/IRS data after dereddening to those found in the original data. Generally it was found that the PAH band intensity variations persist even after dereddening, implying that extinction is not the main cause of the PAH band intensity variations.

The Life Cycle of Dust in the Universe: Observations, Theory, and Laboratory Experiments 18-22 November, 2013

Taipei, Taiwan

\footnotetext{
* Speaker.
} 


\section{Introduction}

In recent years it has been suggested that the observed intensity variations of the polycyclic aromatic hydrocarbon (PAH) bands which dominate mid-infrared (MIR) spectra are generated by extinction, rather than the physical properties of the emitting PAH population [1]. Such variations are commonly detected in most astrophysical contexts and found to be remarkably consistent within and between objects (e.g. [凹]). Moreover, the ubiquity of both the variations in PAH intensities and presence of extinction makes it attractive to suggest that they may be linked.

Here we measure spatial variations in the extinction for the ultra compact HII region IRAS 12063-6259 (hereafter IR12063) in several different ways. These extinction maps were then used to deredden a Spitzer/IRS spectral cube of observations of IRAS 12063-6259. The PAH bands, and correlations, were then measured in both the observed and dereddened cubes. In addition we compared the resulting extinction maps and found unprecedented new behaviour. Full details can be found in the full article [8].

\section{Extinction Variations in IRAS 12063-6259}

In this work we first measured extinction by comparing the fluxes of hydrogen recombination lines to that predicted by case $\mathrm{B}$ recombination theory [5]. Observations of Paschen $\beta$ and Brackett $\gamma$ were obtained using the ISAAC instrument on the VLT for this purpose. Independently of this measurement, the depth of the silicate absorption feature in the Spitzer/IRS cube was measured using an adapted version of the Spoon method (Figure 1, left; [7]). A third map was constructed using recombination lines and radio continuum measurements which we will not discuss further here.

In Figure 1 (right), we show the relationship between extinction as measured in the two different ways. It is clear that the silicate absorption varies significantly across IR12063, while the NIR extinction remains approximately the same. The surrounding regions of IR12063 (labelled 'IR12063 - diffuse' in Figure 1 right) behave as expected, following the diffuse ISM (dISM) relationship [3]. The central regions of IR12063 (labelled 'IR12063 - radio B') show a very different level of silicate absorption (near zero) while retaining the high near-IR extinction. Variations in the relationship between these quantities have been observed before (e.g. the dISM and MC tracks in Figure 1, right) but not for HII regions. In IR12063 the relationship between silicate absorption and NIR extinction varies more on scales of a few parsecs than the observed variation between separate molecular cloud and dISM sightlines.

\section{PAH Correlations for IRAS 12063-6259}

Here we compare the observed PAH variation correlations in a) the data as observed (i.e. affected by extinction), b) the data dereddened using the NIR extinction map and c) the data dereddened using the Spoon method extinction map. In each case the dereddening was performed using the extinction law given by [2]. These data, in the form of correlation plots, are shown in Figure 2 .

The first correlation considered, 7.7/11.2 vs 6.2/11.2, is strongly affected by extinction. Strong silicate extinction preferentially attenuates the $11.2 \mu \mathrm{m}$ band while the 6.2 and $7.7 \mu \mathrm{m}$ bands are 

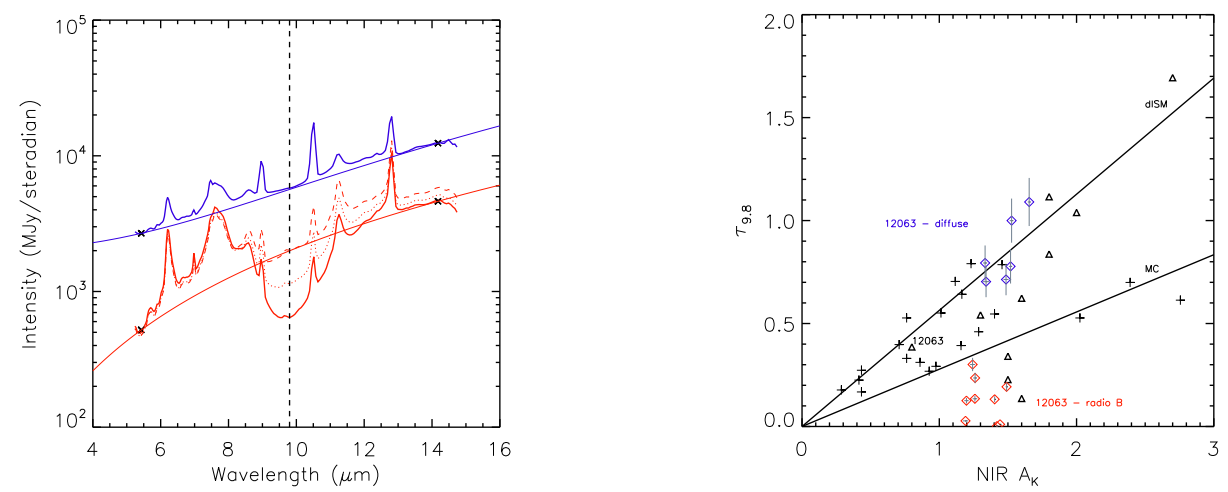

Figure 1: Left: Typical MIR spectra for IR12063. The red and blue lines represent high and low extinction regions respectively. The thin straight lines represent power law continua for each spectrum to which the observed spectrum is compared to find the extinction (the Spoon method). The red dotted and dashed lines show the spectra dereddened using the [2] extinction law before (dotted) and after (dashed) our modification of the Spoon method. Right: The relationship between NIR extinction and silicate absorption at $9.8 \mu \mathrm{m}$. In black we have included trends found for the diffuse ISM (dISM) and molecular cloud material (MC) given by [3], along with their data points as black crosses. Points from the surroundings and core of IR12063 are shown in blue and red respectively, the spectra shown in Figure 1 are representative of these regions (colours reversed). A selection of ISO spectra of UC HII regions drawn from [6] were measured in the same way are shown as black triangles.
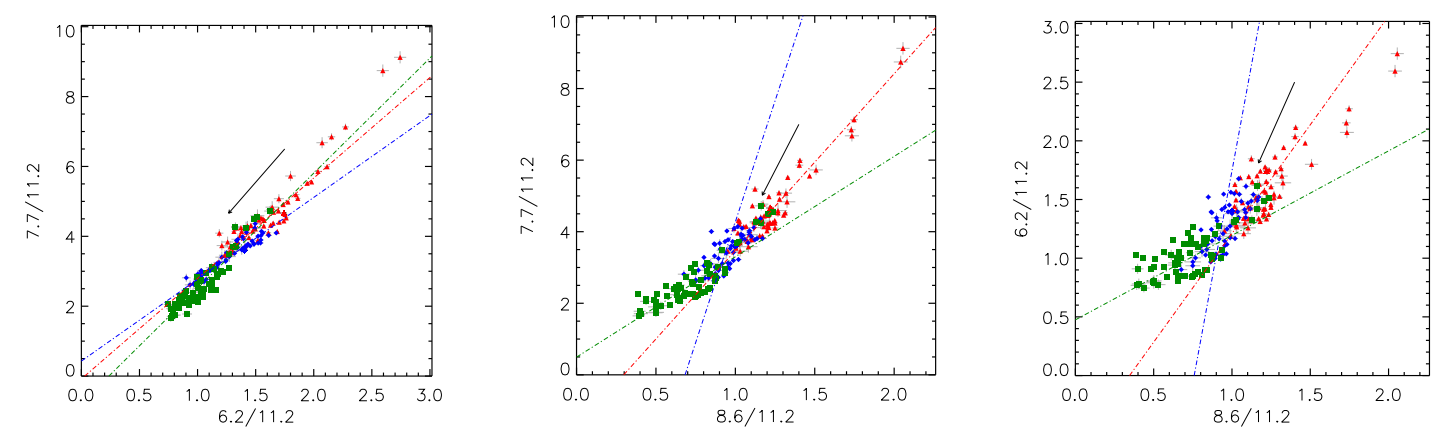

Figure 2: Correlation plots for PAH emission in IR12063. The red, green and blue points represent the non extinction corrected data, the data corrected using the NIR extinction map and the data corrected using the silicate extinction map respectively. The dashed lines represent the best fit slopes for each correlation and the black arrow is a dereddening vector representing $\mathrm{A}_{K}=1$.

affected equally, which stretches the correlation equally in $x$ and $y$ (i.e. the extinction vector shown). Coincidentally for $7.7 / 11.2$ vs $6.2 / 11.2$, the gradient of the extinction vector closely matches that of the correlation, so increased extinction amplifies the correlation coefficients. This also leads to a situation where uncertainties on the extinction measurements do not significantly affect the correlation, as the variations are parallel to the correlation.

In contrast, the other two correlation plots involve the $8.6 \mu \mathrm{m}$ band - which is affected by silicate absorption almost as much as the $11.2 \mu \mathrm{m}$ band. For both 7.7/11.2 vs 8.6/11.2 and 6.2/11.2 vs 8.6/11.2 the correlations are much more scattered for both the observed and dereddened data. This is because the numerator bands are not evenly affected by silicate absorption as in 7.7/11.2 vs 6.2/11.2. The differences in measured extinction then lead to reduced correlation coefficients and 
wildly varying slopes. Moreover, the two different extinction measures actually seem to change the slopes in opposite ways.

\section{Conclusions}

1. Silicate and NIR extinction are spatially decoupled across IR12063,

2. extinction can affect all of the PAH band correlations, but preferentially those involving the 8.6 and $11.2 \mu \mathrm{m}$ bands,

3. for the 6.2 vs 8.6 and 7.7 vs 8.6 correlations, dereddening with different extinction measurements changes the derived correlation slopes in opposite ways!

4. After extinction corrections, PAH correlations persist: extinction is not the main cause of PAH intensity correlations.

5. However, the 6.2 vs 7.7 correlation is actually improved by extinction, because the extinction vector is parallel to the correlation.

\section{Acknowledgments}

DJS and EP acknowledge support from an NSERC Discovery Grant and an NSERC Discovery Accelerator Grant. Studies of interstellar chemistry at Leiden Observatory are supported through advanced-ERC grant 246976 from the European Research Council, through a grant by the Dutch Science Agency, NWO, as part of the Dutch Astrochemistry Network, and through the Spinoza premie from the Dutch Science Agency, NWO.

\section{References}

[1] Beirão, P., et al., Spatially Resolved Spitzer IRS Spectroscopy of the Central Region of M82, 2008, ApJ, 676, 304

[2] Chiar, J. E., \& Tielens, A. G. G. M., Pixie Dust: The Silicate Features in the Diffuse Interstellar Medium, 2006, ApJ, 637, 774

[3] Chiar, J. E., et al., Ices in the Quiescent IC 5146 Dense Cloud, 2011, ApJ, 731, 9

[4] Galliano, F., Madden, S. C., Tielens, A. G. G. M., Peeters, E., \& Jones, A. P., Variations of the Mid-IR Aromatic Features inside and among Galaxies, 2008, ApJ, 679, 310

[5] Hummer, D. G., \& Storey, P. J., Recombination-line intensities for hydrogenic ions. I - Case B calculations for H I and He II, 1987, MNRAS, 224, 801

[6] Peeters, E., et al., ISO spectroscopy of compact H II regions in the Galaxy. I. The catalogue, 2002, A\&A, 381, 571

[7] Spoon, H. W. W., Marshall, J. A., Houck, J. R., Elitzur, M., Hao, L., Armus, L., Brandl, B. R., \& Charmandaris, V., Mid-Infrared Galaxy Classification Based on Silicate Obscuration and PAH Equivalent Width, 2007, ApJL, 654, L49

[8] Stock, D. J., Peeters, E., Tielens, A. G. G. M., Otaguro, J. N., \& Bik, A., Extinction and Polycyclic Aromatic Hydrocarbon Intensity Variations across the H II Region IRAS 12063-6259, 2013, ApJ, 771, 72 